\title{
Necdin Promotes Tangential Migration of Neocortical Interneurons from Basal Forebrain
}

\author{
Takaaki Kuwajima, ${ }^{*}$ Koichi Hasegawa, ${ }^{*}$ and Kazuaki Yoshikawa \\ Laboratory of Regulation of Neuronal Development, Institute for Protein Research, Osaka University, Suita, Osaka 565-0871, Japan
}

Necdin is a pleiotropic protein that promotes neuronal differentiation and survival. In mammals, the necdin gene on the maternal chromosome is silenced by genomic imprinting, and only the paternal necdin gene is expressed in virtually all postmitotic neurons. Necdin forms a complex with the homeodomain protein Dlx2 to enhance its transcriptional activity. Dlx2 plays a major role in controlling tangential migration of GABAergic interneurons from the basal forebrain to the neocortex. Here, we examined whether Dlx2-expressing interneurons migrate properly in vivo in mutant mice lacking the paternal necdin gene. In necdin-deficient mice at birth, the population of Dlx2-expressing cells significantly decreased in the neocortex but increased in the preoptic area. DiI-labeled cell migration assay using organotypic forebrain slice cultures revealed that the number of cells migrating from the medial ganglionic eminence into the neocortex was significantly reduced in necdin-deficient embryos. Furthermore, necdin-deficient mice had a decreased population of neocortical GABA-containing neurons and were highly susceptible to pentylenetetrazole-induced seizures. These results suggest that necdin promotes tangential migration of neocortical GABAergic interneurons during mammalian forebrain development.

\section{Introduction}

Necdin is a neural differentiation-specific protein expressed in murine embryonal carcinoma cells (Maruyama et al., 1991). The necdin gene $(\mathrm{Ndn})$ is expressed in virtually all postmitotic neurons from early development to adult age (Uetsuki et al., 1996). Necdin is a member of melanoma antigen (MAGE) family proteins, all of which contain a conserved MAGE homology domain (MHD) (Barker and Salehi, 2002). Via the MHD, necdin interacts with many regulatory proteins involved in cell proliferation, apoptosis, and differentiation (Hasegawa and Yoshikawa, 2008). Through these interactions, necdin promotes terminal differentiation and survival of various neurons.

Necdin forms a complex, via MAGE-D1, with Msx/Dlx family proteins, which are homeodomain proteins that govern cell fates and differentiation (Kuwajima et al., 2004, 2006). Dlx family proteins play major roles in differentiation and migration of GABAergic interneurons originating in the ventral telencephalon (Marin and Rubenstein, 2003). Mutant mice defective in Dlx1/ Dlx2 homeodomain proteins show abnormal migration of neocortical interneurons from the ventral telencephalon (Anderson et al., 1997b). Together, these findings raise the possibility that necdin promotes tangential migration of Dlx2-expressing cortical interneurons.

Received Nov. 23, 2009; revised Jan. 6, 2010; accepted Feb. 2, 2010.

This work was supported by a Grant-in-Aid for Scientific Research B2 (21300138) from the Japan Society for the Promotion of Science and by the National Project on Protein Structure and Functional Analysis of the Ministry of Education, Culture, Sports, Science, and Technology of Japan.

${ }^{*}$ T.K. and K.H. contributed equally to this work.

Correspondence should be addressed to Kazuaki Yoshikawa, Institute for Protein Research, 0saka University, 3-2 Yamadaoka, Suita, 0saka 565-0871, Japan. E-mail: yoshikaw@protein.osaka-u.ac.jp.

T. Kuwajima's present address: Department of Pathology and Cell Biology, Columbia University, New York, NY 10032.

DOI:10.1523/JNEUROSCI.5797-09.2010

Copyright $\odot 2010$ the authors $\quad 0270-6474 / 10 / 303709-06 \$ 15.00 / 0$
Genomic imprinting is an epigenetic mechanism by which certain genes are silenced in a parent-of-origin-specific manner. Although genomic imprinting is thought to be involved in embryonic growth and development of placental mammals, it remains unclear whether this phenomenon is involved in mammalian brain development. Human necdin gene (NDN) is located on chromosome 15q11-q13, a region responsible for the genomic imprinting-associated neurodevelopmental disease Prader-Willi syndrome (PWS) (Jay et al., 1997; MacDonald and Wevrick, 1997; Nakada et al., 1998). Mutant mice defective in paternal $N d n(p N d n)$ show various types of neuronal abnormalities, including those seen in PWS (Gerard et al., 1999; Muscatelli et al., 2000; Kuwako et al., 2005). Although it is as yet unknown how much necdin deficiency contributes to the pathogenesis of PWS, it is of interest to analyze the phenotypes of necdindeficient mice by comparison with those of PWS.

In the present study, we quantified Dlx2-expressing neurons in the neocortex and preoptic area of $p N d n$-deficient mice. We demonstrate that necdin deficiency reduces the migratory activity of fluorescent dye-labeled cells from the basal forebrain to the neocortex. We also show that the neocortical GABA system is impaired in $p N d n$-deficient mice, which show a high susceptibility to the $\mathrm{GABA}_{\mathrm{A}}$ receptor antagonist pentylenetetrazole (PTZ). Based on these findings, we propose that necdin evolved to promote the development of neocortical GABAergic neurons in mammals.

\section{Materials and Methods}

Animals. Generation, breeding, and genotyping of necdin-deficient mice $\left(\mathrm{Ndn}{ }^{\mathrm{tm} 1 \mathrm{Ky}}\right)$ were described previously (Kuwako et al., 2005). Heterozygous males $\left(\mathrm{Ndn} n^{+-}\right)(>15$ generations in ICR background) were crossed with wild-type ICR females (SLC) to obtain wild-type $\left(\mathrm{Ndn}{ }^{+/+}\right.$) and $p N d n$-deficient $\left(N d n^{+\mathrm{m} /-\mathrm{p}}\right)$ littermates. Experiments using genetargeted mice were approved by the Recombinant DNA and Animal 
Experiment Committees of the Institute for Protein Research, Osaka University (Suita, Japan) and performed in accordance with institutional guidelines and regulations.

Western blotting. Proteins ( $10 \mu \mathrm{g} / \mathrm{lane})$ were separated by $10 \%$ SDS-PAGE, blotted, and detected with antibodies against necdin (NC243; 1:3000) (Niinobe et al., 2000) and $\beta$-tubulin (TUB2.1; Sigma-Aldrich) as described previously (Kuwajima et al., 2006).

Immunohistochemistry. Brain tissues were fixed with $4 \%$ paraformaldehyde solution in $0.1 \mathrm{M}$ phosphate buffer, $\mathrm{pH} 7.4$, overnight and cryoprotected by immersion in 30\% sucrose overnight. Frozen, $12-\mu \mathrm{m}$-thick coronal brain sections at the rostral and caudal positions were prepared, and five sections with close morphological similarities between wild-type and $p N d n$-deficient mice were selected. The sections were incubated overnight or $36 \mathrm{~h}$ with primary antibodies at $4^{\circ} \mathrm{C}$ and fluorescence dye-conjugated secondary antibodies at room temperature for $90 \mathrm{~min}$. Primary antibodies used are anti-Dlx2 (GDlx2; 1:3000) (Kuwajima et al., 2006), anti-GABA (1:3000; Sigma-Aldrich), and anti-necdin (NC243; 1:1000). The secondary antibodies fluorescein isothiocyanateconjugated anti-rabbit IgG (1:500; Cappel), cyanine 3-conjugated anti-guinea pig IgG (1: $500)$, cyanine 2-conjugated anti-guinea pig IgG (1:500), cyanine 3-conjugated anti-mouse IgG (1:500), and cyanine 3-conjugated anti-rabbit IgG (1:500) are from Jackson ImmunoResearch Laboratories. Chromosomal DNA was detected with $3.3 \mu \mathrm{m}$ Hoechst 33342 (Sigma-Aldrich). The images were observed with a fluorescence microscope (BX50-34-FLAD1; Olympus) and taken by CCD camera system (M-3204C; Olympus). For quantification of Dlx2- and GABAcontaining cells, the immunopositive cells per $200-\mu \mathrm{m}$-wide radial column of the neocortical areas were counted.

Cell migration assay. Organotypic forebrain slice cultures were prepared from mouse embryos as described previously (Anderson et al., 1997a; Kuwajima et al., 2006). Selected 300- $\mu \mathrm{m}$ thick sections containing lateral ganglionic eminence (LGE), medial ganglionic eminence (MGE), caudal ganglionic eminence (CGE), or preoptic area (POA) were placed on a collagencoated culture membrane (diameter, $12 \mathrm{~mm}$; pore size, $3.0 \mu \mathrm{m}$; Coaster, Corning), incubated with DMEM/F12 medium containing 10\% fetal calf serum for $30 \mathrm{~min}$, and cultured with Neurobasal medium supplemented with B27 supplement (Invitrogen) and $2 \mathrm{~mm} \mathrm{~L}$-glutamine in a humidified $5 \% \mathrm{CO}_{2}$ incubator at $37^{\circ} \mathrm{C}$ for $48 \mathrm{~h}$. To examine the migratory pathways of neurons generated in the ganglionic eminences (GEs) and POA, similar-sized crystals $(\sim 100 \mu \mathrm{m})$ of DiI $\left(1,1^{\prime}\right.$-dyoctadecyl-3,3,3'.3' -tetramethylindocarbocyanine; Invitrogen) were set on the slices using an insect pin and a dissection microscope. After $48 \mathrm{~h}$ incubation, the slices were fixed in $4 \%$ paraformaldehyde, stained with Hoechst 33342, and observed with the fluorescence microscope using rhodamine fluorescence filters.

PTZ-induced seizures. PTZ-induced seizures were induced and scored according to the method described previously (Tornberg et al., 2005).
A

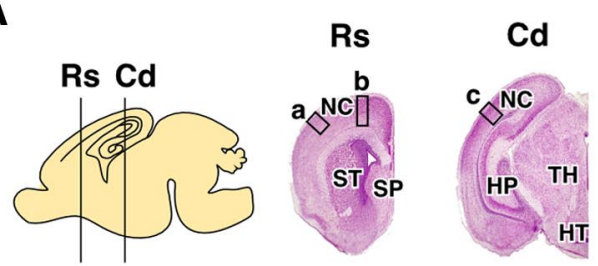

B

BO E14.5

C

pNdn+

pNdn-

Rs
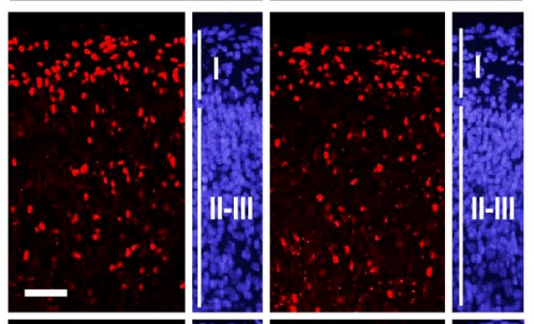

Cd
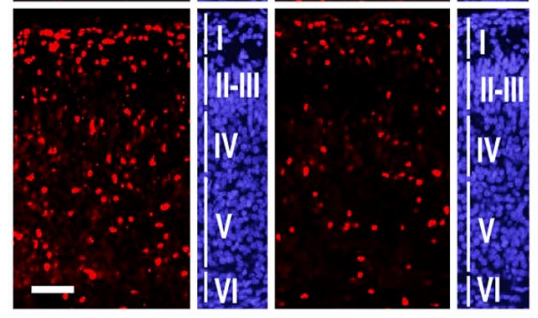

E

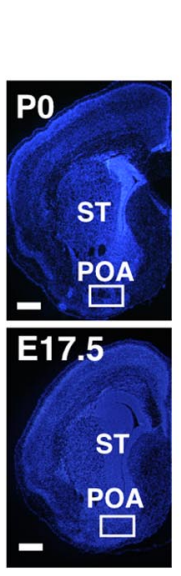

$\mathbf{F}$

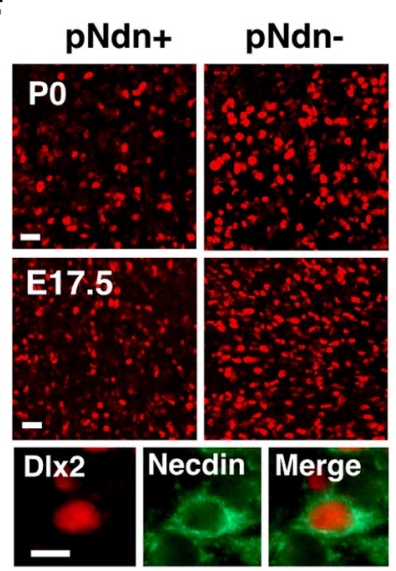

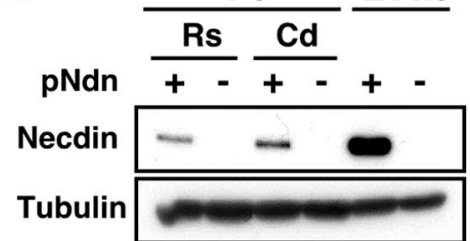

D

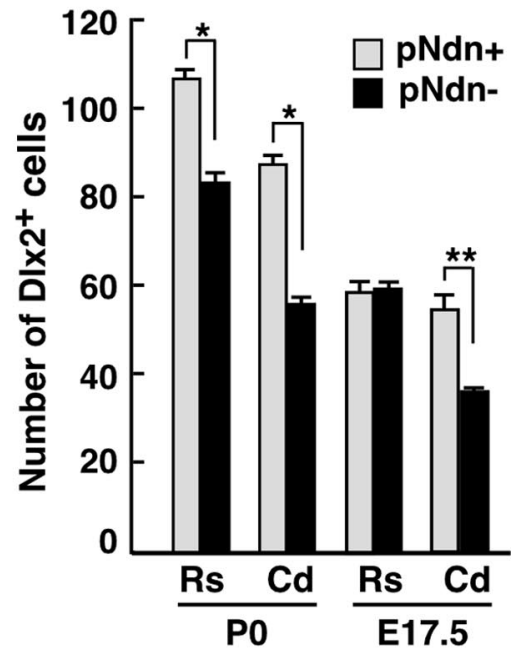

G

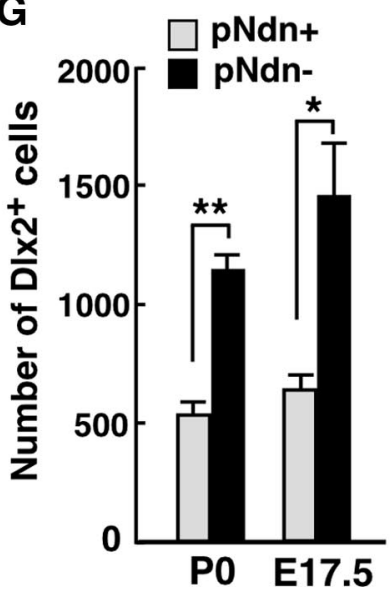

Figure 1. Immunohistochemical analysis of Dlx2-expressing cells in the neocortex and preoptic area. $A$, Coronal sections of the cerebral cortex. Niss-stained sections of PO mouse forebrain at rostral and caudal positions are shown schematically. Boxes a - care the areas analyzed. Rs, Rostral; Cd, caudal; NC, neocortex; SP, septum; ST, striatum; HP, hippocampus; TH, thalamus; HT; hypothalamus. $\boldsymbol{B}$, Western blot analysis. Expression levels of necdin in the rostral (Rs) and caudal (Cd) neocortices at P0 and forebrain at E14.5 of wild-type (pNdn +) and paternal Ndn-deficient ( $\mathrm{pNdn}-$ ) mice were analyzed. Necdin ( $43 \mathrm{kDa}$ ) and $\beta$-tubulin (52 kDa) bands are shown. C, Dlx2 immunostaining patterns in the neocortex. Dlx2-immunopositive cells (red) in the neocortex at the rostral (Rs) and caudal (Cd) positions (boxes a and c, respectively, in $A$ ) of pNdn + and pNdn - mice at PO are shown. The cortical layers were judged by staining DNA (blue). Scale bars, $50 \mu \mathrm{m}$. D, Quantification of Dlx2-expressing cells. Dlx2-immunopositive (Dlx2 ${ }^{+}$) cells in cortical layers $\mathrm{I}-\mathrm{VI}$ were counted and presented as the number per 200- $\mu \mathrm{m}$-wide radial column (examined 5 sections per embryo, mean $\pm \mathrm{SEM}, n=5$ ); ${ }^{*} p<0.05,{ }^{* *} p<0.001$. $E$, Coronal sections of $\mathrm{P} 0$ and E17.5 mouse rostral forebrains. Sections stained for DNA are shown schematically. Scale bars, $500 \mu \mathrm{m}$. $F$, Dlx2 immunostaining patterns in POA. Dlx2-immunopositive cells (red) in a ventrolateral POA region (boxed in $\boldsymbol{E}$ ) of $\mathrm{pNdn}+$ and pNdn - mice at PO (top panels) and E17.5 (middle panels) are shown. A POA section of PO mouse was double-immunostained for Dlx2 and necdin (bottom panels). Scale bars, $20 \mu \mathrm{m} . \mathbf{G}$, Quantification of Dlx2-expressing cells in P0A. Dlx2-immunopositive (Dlx2 ${ }^{+}$) cells in POA (observed area, $200 \times 200 \mu \mathrm{m}$ ) of $\mathrm{pNdn}+$ and $\mathrm{pNdn}-$ mice at $\mathrm{PO}$ and $\mathrm{E} 17.5$ were counted and presented as the number per square millimeter (examined 5 sections per animal, mean \pm SEM, $n=3$ ); ${ }^{*} p<0.05,{ }^{* *} p<0.01$.

Male wild-type $(n=5)$ and necdin-deficient $(n=9)$ mice (P120-150) were injected with PTZ (Sigma-Aldrich) intraperitoneally at a dose of 65 $\mathrm{mg} / \mathrm{kg}$. At this dose, mice showed 100\% seizure frequency. Each mouse was observed for $20 \mathrm{~min}$ after PTZ injection. Lethality (percentage) was 
A

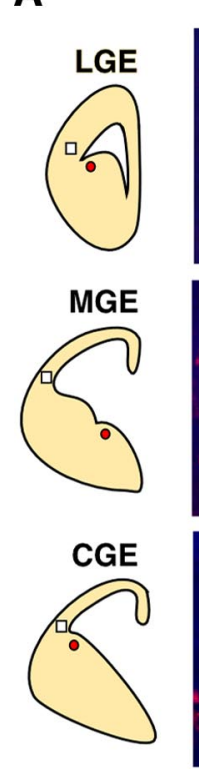

C

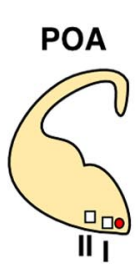

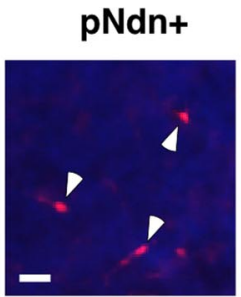
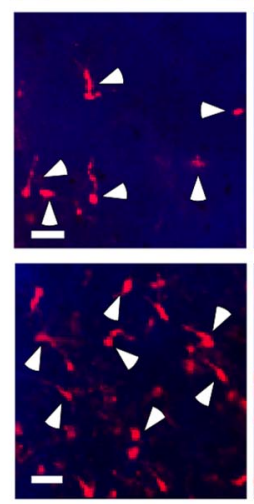

pNdn+

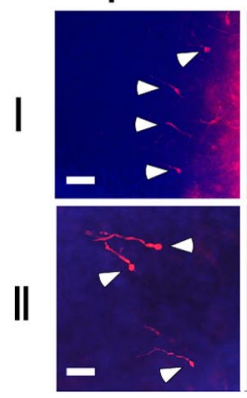

pNdn-
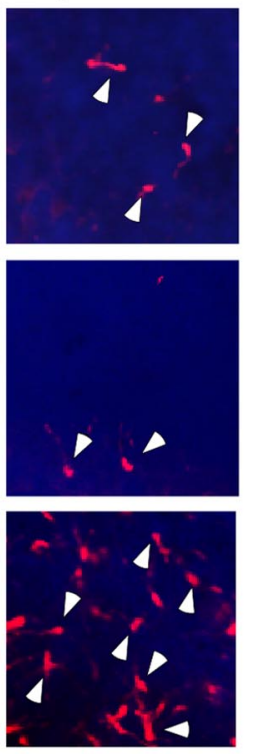

pNdn-

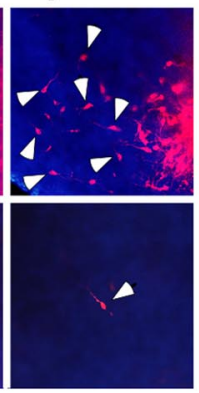

B

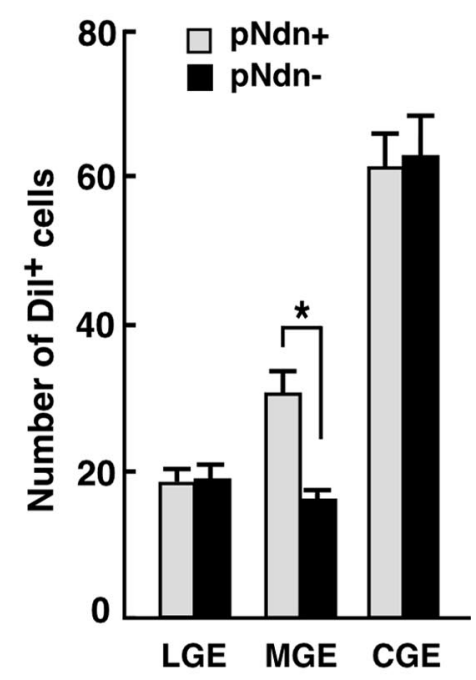

D

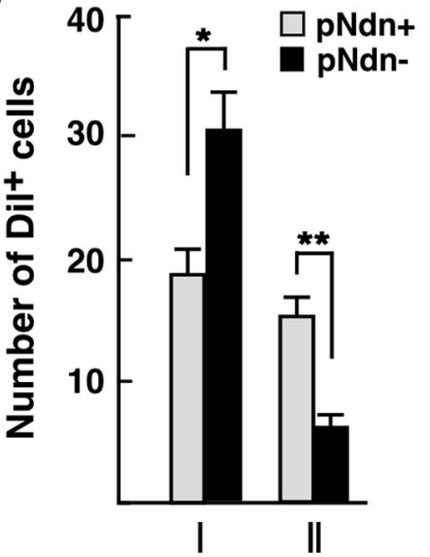

Figure 2. Tangential migration assay. $\boldsymbol{A}$, Tangential migration of Dil-labeled cells to the neocortex. Organotypic slice cultures containing lateral (LGE) (top), medial (MGE) (middle), and caudal (CGE) (bottom) ganglionic eminences were prepared from $\mathrm{pNdn}+$ and pNdn - mice at E14.5. Dil crystals were set on the above areas, and Dil-positive cells were observed $48 \mathrm{~h}$ later. Schemes on the left show the positions of Dil crystals (red circles) and observed areas (white squares). Arrowheads indicate representative Dil-labeled cells. Scale bars, $20 \mu \mathrm{m}$. B, Quantification of Dil-labeled cells in the cortex. Dil-positive (Dil ${ }^{+}$) cells in the observed areas were counted and presented as the total number per $500-\mu \mathrm{m}$-wide radial column (mean $\pm \mathrm{SEM}, n=10$ ); ${ }^{*} p<$ 0.001. C, Tangential migration of Dil-labeled cells in the POA. Dil crystals were set on the POA in organotypic slices prepared from pNdn + and pNdn - mice atE14.5 and cultured for $48 \mathrm{~h}$. Dil-labeled cells in areas I and II (white squares) at $\sim 200$ and $\sim 600 \mu \mathrm{m}$, respectively, distant from Dil crystals (red circle) were observed. Scale bars, $20 \mu \mathrm{m}$. Arrowheads point to representative Dil-labeled cells. D, Quantification of Dil-labeled cells in POA. Dil-labeled (Dil $\left.{ }^{+}\right)$cells in observed areas I and II (100 $\times 100 \mu \mathrm{m}$ each) were counted and presented as the number per observed area (examined 3 nonoverlapping areas per slice, mean \pm SEM, $n=16$ ); ${ }^{*} p<$ $0.05,{ }^{* *} p<0.01$.

calculated from the number of dead mice during the test period. The seizure frequency and lethality were calculated as the percentage of mice in each group with seizures scored 3. Latency to the onset of the first clonic seizure was recorded, and durations of clonic seizures during the test period in each mouse were averaged. Data were presented as mean \pm SEM. Mice to which the first seizure was lethal were excluded from the data.

Statistical tests. Statistical significance was tested using an unpaired Student $t$ test or one-way ANOVA followed by Tukey's post hoc test. A significance of $p<0.05$ was required for rejection of the null hypothesis. For analysis of lethality after PTZ-induced convulsions, Fisher's exact probability test was used.

\section{Results}

We first analyzed the expression levels of necdin in the rostral and caudal neocortices of wild-type and $p N d n$-deficient littermates at postnatal day $0(\mathrm{P} 0)$ by Western blot analysis (Fig. $1 A, B$ ). Necdin was detectable in these areas of wild-type mice, although the levels were much lower in the neocortex than in the forebrain at embryonic day 14.5 (E14.5). Necdin was totally undetectable in $p N d n$-deficient mice. We next quantified the Dlx2-expressing cells in the neocortex by immunohistochemistry and found that the number of Dlx2immunopositive cells was significantly reduced in $p N d n$-deficient mice (Fig. $1 C, D)$. The number of Dlx2-expressing cells was more reduced (36\% reduction) in the caudal cortex than in the rostral cortex $(22 \%$ reduction). In particular, $p N d n$ deficient mice exhibited a marked reduction in the number of Dlx2-immunopositive cells in layers I-III of the caudal cortex (56\% of the wild-type control) (wild type, $45.4 \pm$ 1.8; $p N d n$ deficient, $25.2 \pm 0.4: p<0.001$ ). On the other hand, no appreciable change in the cortical population of glutamic acidcontaining neurons was noted between wild-type and $p N d n$-deficient mice at P0 (data not shown), suggesting that cortical Dlx2-dependent neurons originating from the ventral telencephalon are specifically affected in $p N d n$-deficient mice.

We then examined when the reduction of neocortical interneurons expressing Dlx2 becomes evident in $p N d n$-deficient mice. At E17.5, there was a significant reduction in the number of Dlx2-expressing cells in the caudal neocortex in $\mathrm{pNdn-}$ deficient mice ( $34 \%$ reduction). In contrast, the number of Dlx2-expressing neurons was unchanged in the rostral neocortex of $p N d n$-deficient mice (Fig. 1D), suggesting that the abnormal distribution of lateborn interneurons, which are still migrating at E17.5, becomes apparent in this region at $\mathrm{P} 0$. At E13.5, there was no difference in the number of Dlx2-positive cells in the marginal-to-intermediate zone between the wild-type and $p N d n$-deficient mice (wild type, $25.0 \pm 1.4 ; p N d n$ deficient, $25.9 \pm 0.7: p=0.58)$, suggesting that the migration of Dlx2-expressing neurons that are born later than E13.5 is impaired in $p N d n$ deficient mice.

We have previously found that cells expressing high levels of Dlx2 are distributed in the ventral telencephalon, including the GEs and POA (Kuwajima et al., 2006). Thus, we quantified Dlx2expressing cells in the POA as a control (Fig. $1 E$ ). We counted the Dlx2-immunopositive cells in a ventrolateral part of the POA in $p N d n$-deficient and wild-type littermates at P0 and E17.5 (Fig. $1 F)$. Remarkably, the numbers of Dlx2-expressing cells in $p N d n$ deficient mice were 2.0 and 2.2 times those of wild-type controls in this region at $\mathrm{P} 0$ and $\mathrm{E} 17.5$, respectively (Fig. $1 G$ ). These results suggest that Dlx2-expressing neuron migration via the POA is impaired in necdin-deficient mice, leading to their accumulation in this area. 
Most of the cortical interneurons are derived from the GEs during the embryonic period (Marin and Rubenstein, 2003). We then quantified DiI-labeled neurons migrating from the GEs to the cerebral cortex (Fig. 2A,B). DiI crystals were set on the specific points in E14.5 forebrain slices, and DiI-labeled cells that migrated into basal neocortical areas were quantified $48 \mathrm{~h}$ later (Fig. $2 \mathrm{~A}$ ). The number of DiI-labeled cells migrating from the MGE was significantly reduced in $p N d n$ deficient mice (48\% reduction), whereas there was no appreciable difference in the number of DiI-labeled cells migrating from the LGE or the CGE between $p N d n$ deficient and wild-type mice (Fig. 2B), suggesting that necdin promotes the tangential migration of MGE-derived interneurons during this period. In this assay, we failed to evaluate the accumulation of DiI-labeled, MGE-derived interneurons within the MGE of $p N d n$-deficient mice.

We also examined the migratory activity of the cells located in the POA at this stage. We quantified DiI-labeled neurons present in the proximal and distal areas at $\sim 200 \mu \mathrm{m}$ (area I) and $\sim 600 \mu \mathrm{m}$ (area II), respectively, distant from the DiI spots in the POA (Fig. 2C). The number of DiIlabeled cells in area I of $p N d n$-deficient mice was 1.6 times that of the wild-type control, whereas the number of DiI-labeled cells in area II of $p N d n$-deficient mice was $38 \%$ of the control number (Fig. 2D). These results suggest that necdin deficiency impairs tangential migration of neurons, resulting in their accumulation in the POA on the route to their destinations.

$D l \times 2$ is a master regulatory gene that induces differentiation of cortical GABAergic interneurons. We thus examined the population of GABA-containing cells in the neocortex (motor cortex) (Fig. 3A). We found that the number of intensely GABAimmunopositive neurons in this area of $p N d n$-deficient mice was significantly reduced to $59 \%$ of the wild-type control number (wild type, $11.9 \pm 0.8 ; p N d n$ deficient, $7.0 \pm 0.5 ; p<0.01)$. Most of the intensely GABA-immunopositive neurons were positive for Dlx2 in the nucleus. The necdin immunoreactivity was distributed throughout the neocortical layers in wild-type mice. GABA-containing neurons overlapped with Dlx2- and necdin-expressing neurons (Fig. 3B). The Dlx2 immunoreactivity was localized in the nucleus, whereas the GABA and necdin immunoreactivities were detected predominantly in the cytoplasm.

Neocortical GABAergic interneurons form inhibitory circuits to suppress the excitation of glutamatergic neurons. Thus, we examined whether $p N d n$-deficient mice show a higher susceptibility to $\mathrm{GABA}_{\mathrm{A}}$ receptor antagonist PTZ than wild-type mice. There was no significant difference in the lethality between wild-
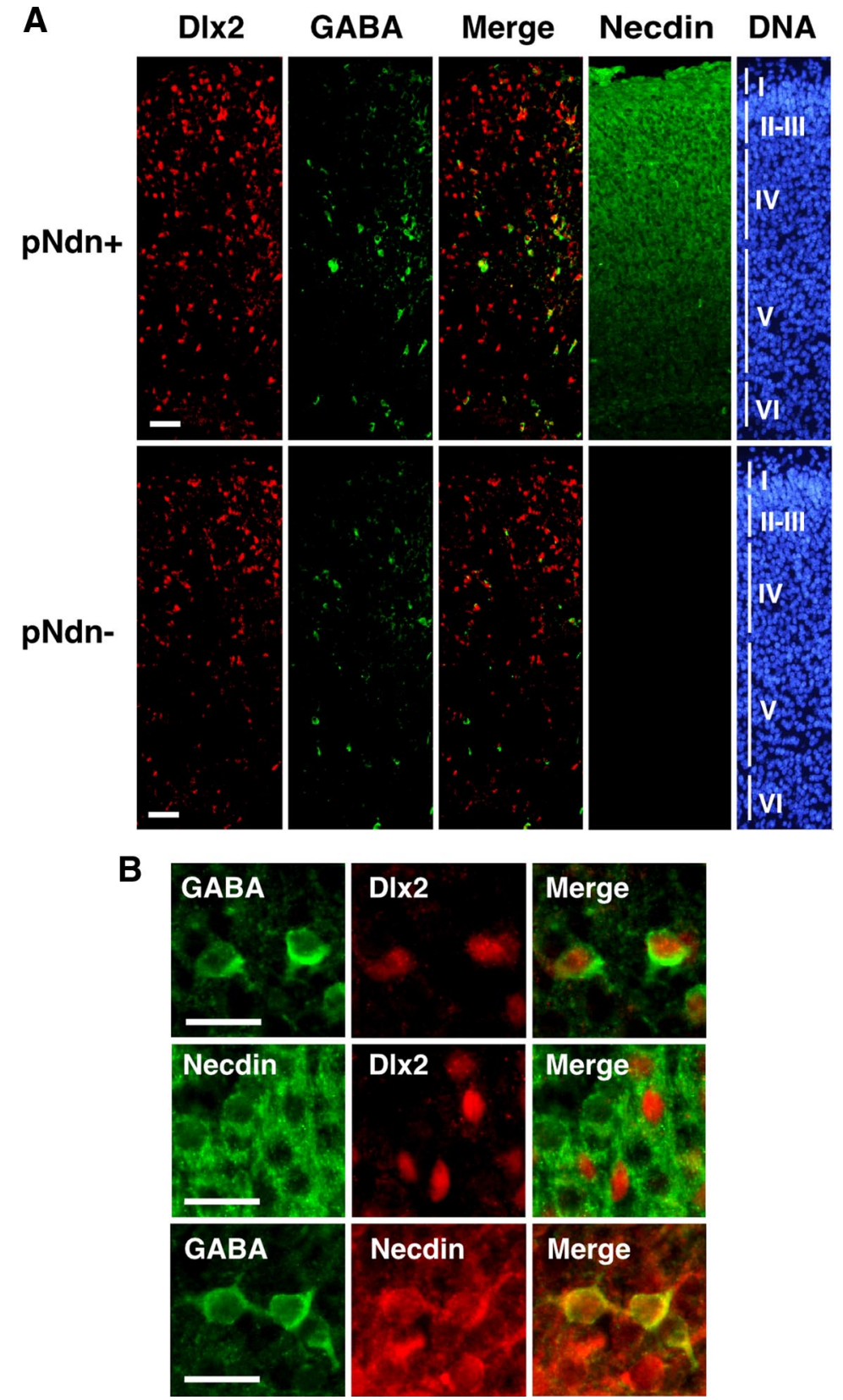

Figure 3. Immunohistochemical analysis of neocortical GABAergic neurons. $\boldsymbol{A}$, Double immunostaining for DIx2 and GABA. Cells in the rostral neocortex at $\mathrm{PO}$ (shown as box b in Fig. $1 A$ ) of pNdn + and pNdn - mice were immunostained for DIx2 (red) and GABA (green), and two images were merged. Necdin was also immunostained for reference. The cortical layers were judged by staining pNdn + mice at PO were double immunostained for GABA and DIx2 (top panels), necdin and Dlx2 (middle panels), or GABA and necdin (bottom panels). Images in neocortical layer V are shown. Two images are merged for colocalization. Scale bars, $20 \mu \mathrm{m}$.

type controls and $p N d n$-deficient mice (wild type, $4, n=6 ; p N d n$ deficient, $7, n=9: p=0.45$ on Fisher's exact probability test; expected frequency in $\chi^{2}$ test, $\left.<5\right)$. However, the seizure latency in $p N d n$-deficient mice was markedly reduced to $33 \%$ of the wildtype control (wild type, $490.6 \pm 43.0 \mathrm{~s}$; $p N d n$ deficient, $162.6 \pm$ $25.4 \mathrm{~s}: p<0.001$ ). In addition, the seizure duration of $p N d n$ deficient mice was 2.9 times that of the wild-type control (wild type, $15.5 \pm 1.5 \mathrm{~s} ; p N d n$ deficient, $45.0 \pm 7.9 \mathrm{~s}: p<0.02$ ). These data indicate that the inhibitory function of cortical GABAergic interneurons is impaired in $p N d n$-deficient mice, which are hypersensitive to PTZ-induced seizures. To confirm that the neocortical interneuron population remains decreased in 
adulthood, we quantified cortical Dlx2-expressing cells in necdindeficient mice at P120. The number of Dlx2-immunopositive cells in layers I-IV of the $p N d n$-deficient mouse cortex was $69 \%$ that of the wild-type control (wild type, $21.2 \pm 1.5$; $p N d n$ deficient, $14.6 \pm 1.4$ : $p<0.05)$.

\section{Discussion}

The present study has shown that necdin-deficient mice display impaired migration of Dlx2-containing interneurons from the subpallium to the neocortex. Consistent with the fact that cells from the MGE give rise to a majority of cortical GABAergic interneurons (Marin and Rubenstein, 2003), the migratory activities of the MGE cells were specifically reduced in $p N d n$-deficient embryos (Fig. 2 A). These observations suggest that endogenous necdin promotes tangential migration of Dlx2-dependent neurons from the MGE into the neocortex at the embryonic stage. Similarly, tangential migration of gonadotropin-releasing hormone $(\mathrm{GnRH})$ neurons is attenuated from the olfactory epithelium to the anterior hypothalamus in necdin-deficient mouse embryos (Miller et al., 2009). GnRH neuron development is also dependent on Msx/Dlx family proteins (Givens et al., 2005). These findings support the notion that necdin collaborates with Msx/Dlx homeodomain transcription factors to modulate the migratory activities of both neocortical interneurons and $\mathrm{GnRH}$ neurons.

Neuronal apoptosis is significantly increased in the dorsal root ganglia and the superior cervical ganglia of $p N d n$-deficient mice (Kuwako et al., 2005; Tennese et al., 2008). In contrast, necdin deficiency exerts no appreciable effects on the number of Dlx2expressing neurons differentiating both in vivo and in vitro (Kuwajima et al., 2006). Thus, the reduction of neocortical Dlx2expressing interneurons may not be attributable to the increased apoptosis but mainly to the attenuated migration in $p N d n$ deficient mice. Consistent with this idea, the number of Dlx2 neurons was markedly increased in the ventrolateral part of the POA (Fig. $1 F, G$ ) in which migrating DiI-labeled cells are prone to accumulate (Fig. 2C,D). Because a substantial number of cortical GABAergic interneurons originate in the POA (Gelman et al., 2009), the POA-derived cells may accumulate within the POA of $p N d n$-deficient mice. However, it is unlikely that the reduction of neocortical interneurons in necdin-deficient mice is attributable to the abnormal migration of POA-derived cells, which contribute to a relatively small population in the neocortex.

Mice lacking both $D l x 1$ and $D l \times 2$ show no detectable cell migration from the subcortical telencephalon to the neocortex, where few GABA-expressing cells are present (Anderson et al., $1997 b)$. In contrast, the phenotype of $p N d n$-deficient mice is milder than that of the $D l \times 1 / D l \times 2$ double-mutant mice. This may be because necdin potentiates the effects of Dlx 2 only on the differentiation and migration of GABAergic interneurons. Necdin interacts with Dlx2 via MAGE-D1 (also known as NRAGE or Dlxin1), regulates Dlx2-dependent transcriptional activities, and promotes GABAergic neuron differentiation (Kuwajima et al., 2006). Thus, necdin and Dlx 2 may cooperate to transactivate certain genes required for the tangential migration of GABAergic interneurons. For example, Dlx2 upregulates TrkB expression in retinal ganglion cells (de Melo et al., 2008). The TrkB signaling via PI3-kinase (phosphoinositide 3-kinase) activation plays an important role in controlling interneuron migration during development of the cerebral cortex (Polleux et al., 2002). Dlx2 also transcriptionally activates the expression of Arx, another homeodomain transcription factor contributing to the migration of GABAergic interneurons (Colasante et al., 2008). We found that a cortical population of Arx-expressing cells, like that of Dlx2expressing cells, is significantly reduced in necdin-deficient mice (T. Kuwajima and K. Yoshikawa, unpublished observations). Thus, necdin may enhance Dlx2-dependent expression of regulatory genes that are involved in the tangential migration of GABAergic interneurons. We are currently investigating such specific genes that are upregulated through the cooperation between Dlx2 and necdin.

The present study has shown that the neocortical GABAergic system is abnormal in necdin-deficient mice. The GABAergic system is required for preventing hyperexcitation of cortical pyramidal neurons. Although there is little information about neocortical GABAergic system in PWS, it has been reported that the frequency of generalized seizure disorder in PWS patients is significantly higher than in the general pediatric population (Fan et al., 2009). Furthermore, cortical GABAergic interneurons are thought to be involved in the pathogenesis of autism (Rubenstein and Merzenich, 2003). The majority of PWS individuals show a striking autistic-like behavioral phenotype (Descheemaeker et al., 2006). Thus, the present results may account, at least in part, for the mechanisms of such neurobehavioral abnormalities seen in PWS.

More than 25 members of MAGE family genes have been identified in human and mouse genomes (Barker and Salehi, 2002). In view of the evolution of necdin and other MAGE family genes, it is noteworthy that necdin is absent from primitive mammals such as the monotreme platypus and the marsupials tammar wallaby and opossum (Rapkins et al., 2006) and that chicken has only a single MAGE, which is functionally similar to necdin (Lopez-Sanchez et al., 2007). These findings indicate that MAGE family genes were rapidly diversified during the course of mammalian evolution. It is tempting to speculate that necdin coevolved with neocortical interneuron diversification in eutherian brains. The present findings may provide valuable insights into the molecular mechanisms underlying forebrain development in placental mammals.

\section{References}

Anderson SA, Qiu M, Bulfone A, Eisenstat DD, Meneses J, Pedersen R, Rubenstein JL (1997a) Mutations of the homeobox genes Dlx-1 and Dlx-2 disrupt the striatal subventricular zone and differentiation of late born striatal neurons. Neuron 19:27-37.

Anderson SA, Eisenstat DD, Shi L, Rubenstein JL (1997b) Interneuron migration from basal forebrain to neocortex: dependence on Dlx genes. Science 278:474-476.

Barker PA, Salehi A (2002) The MAGE proteins: emerging roles in cell cycle progression, apoptosis, and neurogenetic disease. J Neurosci Res 67:705-712.

Colasante G, Collombat P, Raimondi V, Bonanomi D, Ferrai C, Maira M, Yoshikawa K, Mansouri A, Valtorta F, Rubenstein JL, Broccoli V (2008) Arx is a direct target of Dlx2 and thereby contributes to the tangential migration of GABAergic interneurons. J Neurosci 28:10674-10686.

de Melo J, Zhou QP, Zhang Q, Zhang S, Fonseca M, Wigle JT, Eisenstat DD (2008) Dlx2 homeobox gene transcriptional regulation of Trkb neurotrophin receptor expression during mouse retinal development. Nucleic Acids Res 36:872-884.

Descheemaeker MJ, Govers V, Vermeulen P, Fryns JP (2006) Pervasive developmental disorders in Prader-Willi syndrome: the Leuven experience in 59 subjects and controls. Am J Med Genet A 140:1136-1142.

Fan Z, Greenwood R, Fisher A, Pendyal S, Powell CM (2009) Characteristics and frequency of seizure disorder in 56 patients with Prader-Willi syndrome. Am J Med Genet A 149A:1581-1584.

Gelman DM, Martini FJ, Nobrega-Pereira S, Pierani A, Kessaris N, Marin O (2009) The embryonic preoptic area is a novel source of cortical GABAergic interneurons. J Neurosci 29:9380-9389.

Gerard M, Hernandez L, Wevrick R, Stewart CL (1999) Disruption of the 
mouse necdin gene results in early post-natal lethality. Nat Genet 23:199-202.

Givens ML, Rave-Harel N, Goonewardena VD, Kurotani R, Berdy SE, Swan CH, Rubenstein JL, Robert B, Mellon PL (2005) Developmental regulation of gonadotropin-releasing hormone gene expression by the MSX and DLX homeodomain protein families. J Biol Chem 280:19156-19165.

Hasegawa K, Yoshikawa K (2008) Necdin regulates p53 acetylation via Sirtuin1 to modulate DNA damage response in cortical neurons. J Neurosci 28:8772-8784.

Jay P, Rougeulle C, Massacrier A, Moncla A, Mattei MG, Malzac P, Roeckel N, Taviaux S, Lefranc JL, Cau P, Berta P, Lalande M, Muscatelli F (1997) The human necdin gene, NDN, is maternally imprinted and located in the Prader-Willi syndrome chromosomal region. Nat Genet 17:357-361.

Kuwajima T, Taniura H, Nishimura I, Yoshikawa K (2004) Necdin interacts with the Msx2 homeodomain protein via MAGE-D1 to promote myogenic differentiation of C2C12 cells. J Biol Chem 279:40484-40493.

Kuwajima T, Nishimura I, Yoshikawa K (2006) Necdin promotes GABAergic neuron differentiation in cooperation with Dlx homeodomain proteins. J Neurosci 26:5383-5392.

Kuwako K, Hosokawa A, Nishimura I, Uetsuki T, Yamada M, Nada S, Okada M, Yoshikawa K (2005) Disruption of the paternal necdin gene diminishes TrkA signaling for sensory neuron survival. J Neurosci 25:7090-7099.

Lopez-Sanchez N, Gonzalez-Fernandez Z, Niinobe M, Yoshikawa K, Frade JM (2007) Single mage gene in the chicken genome encodes CMage, a protein with functional similarities to mammalian type II Mage proteins. Physiol Genomics 30:156-171.

MacDonald HR, Wevrick R (1997) The necdin gene is deleted in PraderWilli syndrome and is imprinted in human and mouse. Hum Mol Genet 6:1873-1878.

Marin O, Rubenstein JL (2003) Cell migration in the forebrain. Annu Rev Neurosci 26:441-483.

Maruyama K, Usami M, Aizawa T, Yoshikawa K (1991) A novel brainspecific mRNA encoding nuclear protein (necdin) expressed in neurally differentiated embryonal carcinoma cells. Biochem Biophys Res Commun 178:291-296.

Miller NL, Wevrick R, Mellon PL (2009) Necdin, a Prader-Willi syndrome candidate gene, regulates gonadotropin-releasing hormone neurons during development. Hum Mol Genet 18:248-260.

Muscatelli F, Abrous DN, Massacrier A, Boccaccio I, Le Moal M, Cau P, Cremer H (2000) Disruption of the mouse Necdin gene results in hypothalamic and behavioral alterations reminiscent of the human PraderWilli syndrome. Hum Mol Genet 9:3101-3110.

Nakada Y, Taniura H, Uetsuki T, Inazawa J, Yoshikawa K (1998) The human chromosomal gene for necdin, a neuronal growth suppressor, in the Prader-Willi syndrome deletion region. Gene 213:65-72.

Niinobe M, Koyama K, Yoshikawa K (2000) Cellular and subcellular localization of necdin in fetal and adult mouse brain. Dev Neurosci 22:310-319.

Polleux F, Whitford KL, Dijkhuizen PA, Vitalis T, Ghosh A (2002) Control of cortical interneuron migration by neurotrophins and PI3-kinase signaling. Development 129:3147-3160.

Rapkins RW, Hore T, Smithwick M, Ager E, Pask AJ, Renfree MB, Kohn M, Hameister H, Nicholls RD, Deakin JE, Graves JA (2006) Recent assembly of an imprinted domain from non-imprinted components. PLoS Genet 2:e182.

Rubenstein JL, Merzenich MM (2003) Model of autism: increased ratio of excitation/inhibition in key neural systems. Genes Brain Behav 2:255-267.

Tennese AA, Gee CB, Wevrick R (2008) Loss of the Prader-Willi syndrome protein necdin causes defective migration, axonal outgrowth, and survival of embryonic sympathetic neurons. Dev Dyn 237:1935-1943.

Tornberg J, Voikar V, Savilahti H, Rauvala H, Airaksinen MS (2005) Behavioural phenotypes of hypomorphic KCC2-deficient mice. Eur J Neurosci 21:1327-1337.

Uetsuki T, Takagi K, Sugiura H, Yoshikawa K (1996) Structure and expression of the mouse necdin gene. Identification of a postmitotic neuronrestrictive core promoter. J Biol Chem 271:918-924. 